\title{
Milrinona inhalada en hipertensión pulmonar: a propósito de un caso
}

\author{
Michelle Escobar', Fernando Aranda'
}

Introducción: La hipertensión pulmonar (HTP) es una enfermedad caracterizada por la elevación crónica de las presiones de arteria pulmonar (PAP) y un aumento de la resistencia vascular pulmonar (RVP). La milrinona inhalada ha demostrado actuar como un vasodilatador pulmonar selectivo siendo una herramienta útil en el manejo de los pacientes con HTP en el perioperatorio.

Materiales y Métodos: Reportamos un caso exitoso con uso de milrinona inhalada en HTP en cirugía cardiovascular. La paciente firmó el consentimiento informado para este reporte.

Caso Clínico: Mujer de 62 años, con enfermedad coronaria severa de 3 vasos revascularizada hace 3 meses mediante puentes mamarios a la ADA (LIMA) y a la ACX (RIMA) y un puente venoso a ADP. Cursando en el postoperatorio con ICC descompensada hasta el Edema Pulmonar en dos ocasiones. El Ecocardiograma Transtorácico preoperatorio destaca válvula mitral de velos muy engrosados con apertura normal y disminución de coaptación de los velos con insuficiencia mitral (IM) severa, función ventricular conservada. Cavidades derechas normales con PAP sistólica calculada de $50 \mathrm{mmHg}$. La Coronariografía confirmó solo permeabilidad residual de LIMA. Ingresa para recambio valvular mitral (RVM), realizándose Anestesia General con monitoreo invasivo y Ecocardiografía Transesofágica (ETE). En ETE destaca marcado deterioro de la función VI. En contexto de cardiopatía coronaria se inicia apoyo con Levosimendán 0,1ug/ $\mathrm{kg} / \mathrm{min}$ y Adrenalina $0,01 \mathrm{ug} / \mathrm{kg} / \mathrm{min}$
EV. Tras Esternotomía, la paciente comienza con elevación progresiva de las PAP, agravamiento IM, hipotensión y falla del VD al ETE, sospechándose lesión de LIMA. Debido a Arteriopatía Periférica no fue posible la instalación de Balón de Contrapulsación Aórtico; administrándose $4 \mathrm{ml}$ milrinona al 0,025\% nebulizada en 15 minutos, obteniendo mejoría de los parámetros hemodinámicos y ecocardiográficos permitiendo completar la disección de los planos quirúrgicos y canulación para la CEC. Se realizó puente safeno a LIMA y RVM sin incidentes.

Discusión y Resultados: Pacientes con HTP tienen mayor riesgo de complicaciones perioperatorias (mortalidad hasta 37 - 90\%). Su manejo con vasodilatadores intravenosos es frecuentemente limitado por sus efectos secundarios de vasodilatación e hipotensión, perjudicando la presión de perfusión miocárdica. La Milrinona es un inodilatador que actúa como inhibidor de la fosfodiesterasa III. Wang et al y estudios posteriores han demostrado que administrada por vía inhalatoria actúa como un vasodilatador pulmonar selectivo e inótropo, con menor efecto sistémico.

Conclusión: La Milrinona inhalada ha demostrado ser una herramienta útil para la disminución de la PAP, RVP y mejoría del inotropismo, sin efectos sistémicos deletéreos. Su amplia disponibilidad, menor costo y facilidad de administración hacen pensar que podría ser una herramienta útil para el manejo el perioperatorio de los pacientes con HTP. Hacen falta más trabajos para definir sus potencialidades.

Universidad de Valparaíso, Chile. 